GRADIATION\&APPLICATIONS

ISSN 2466-4294 (online) | rad-journal.org

Vol. 2 | Issue 2 | pp. $101-107,2017$

doi: 10.21175/RadJ.2017.02.022

Original research paper

\title{
IRRADIATION ACTIVITY WITH THE TOP-IMPLART PROTON LINEAR ACCELERATOR*
}

\author{
M. Vadrucci**, A. Ampollini, F. Borgognoni, P. Nenzi, \\ L. Picardi, C. Ronsivalle, V. Surrenti, E. Trinca
}

ENEA - Development of particle accelerators and Medical Applications Laboratory, Fusion and Nuclear Security Department, Physical Technologies for Safety and Health Division, Frascati, Rome, Italy

\begin{abstract}
A proton linear accelerator devoted to proton therapy application, is under construction in the "Particle Accelerators and Medical Applications Laboratory" at the ENEA Frascati research center in the framework of the TOP (Terapia Oncologica con Protoni) - IMPLART (Intensity Modulated Proton Linear Accelerator for RadioTherapy) project funded by the regional government of Lazio in Italy. The proton linac is composed by a modular sequence of RF linear accelerators designed to reach the energy of $15 \mathrm{O} \mathrm{MeV}$. The beam features, particularly useful for very conformal irradiation of tumours in complex anatomical regions, can be likewise translated to other situations. Therefore, during the process of commissioning of the TOP-IMPLART accelerator, the beam has been also made available as a versatile proton source for ancillary experiments in the framework of other projects. Presently, indeed, the maximum TOP-IMPLART beam energy is $35 \mathrm{MeV}$ and this section delivers a 3 usec pulsed beam at the maximum repetition frequency of $25 \mathrm{~Hz}$ with a variable charge in each pulse in the range 5-10o $\mathrm{pC}$. This beam is used for pilot experiments to simulate cosmic conditions on the ground and PIXE (Particle Induced X-ray Emission) analysis for the determination of elemental composition of archeological and old painting samples. This work presents an overview of these activities, describing in detail the different set up adopted to perform the tests and the main achieved results.
\end{abstract}

Key words: Proton irradiation, proton accelerator, PIXE, dosimetric characterization, radiation of plants

\section{INTRODUCTION}

Ionising radiations are conventionally used to treat cancer. The largest medical use of radiations is related to the radiotherapy with high energy photons: the beams of X-rays are produced by accelerated electrons and then delivered to the tumor target from many angles, while trying to spare the surrounding normal tissues. Nevertheless, the accuracy of the irradiation process inevitably leads to the situation in which some radiation dose is always deposited in the healthy tissues.

Conversely, particle beams can penetrate the tissues with little diffusion and deposit the maximum energy just before stopping. Thus, this allows a precise definition of the specific region to be irradiated and a damage to healthy tissues that is lower than in the case of X-rays. Hereof and due to the improvements in accelerator technology, coupled with advances in medical imaging and computing, the use of hadrons in oncological radiotherapy is wider, as the many existing hadrontherapy centers already point out [1].

As of today, all the hadrontherapy centers in operation are based on circular accelerators (cyclotrons and synchrotrons). These facilities imply needfully high development and usage costs, very large sites, large shielding for radiation protection of the primary and passively generated secondary beams, and great power consumption. Therefore, the hadron therapy community well recognizes that hadron sources need further development, especially considering the treatment with protons that has globally started a huge momentum: by 2020 it is expected that there will be almost 100 centres around the world, with over 30 in Europe.

On the basis of its expertise in design and realization of linear particle accelerators [2-5], ENEA has studied an alternative scheme based on a compact high frequency fully linear proton accelerator that offers an optimal solution to the present challenges of proton therapy.

The use of a high frequency implies a more compact and shorter linac than the standard lower-frequency proton linacs used as injectors of most synchrotrons [6].

The ENEA TOP-IMPLART Project was launched in collaboration with the Italian National Institute of Health (ISS) and Regina Elena National Cancer Institute-IFO-Rome and was funded by the

\footnotetext{
* The paper was presented at the Fifth International Conference on Radiation and Applications in Various Fields of Research (RAD 2017), Budva, Montenegro, 2017.

monia.vadrucci@enea.it
} 
M. Vadrucci et al., Irradiation activity..., Rad. Applic., 2017, 2, 2, 101-107

Government of Regione Lazio. The proton accelerator is under construction in the ENEA Frascati research center and consists of a sequence of linear accelerators (Figure 1): a low energy injector composed by a $3 \mathrm{MeV}$ radio frequency quadrupole (RFQ) followed by a $7 \mathrm{MeV}$ drift tube linac (DTL), and by a series of novel structures named side-coupled drift tube linac (SCDTL), invented in 1997 by the InnovationDepartment of ENEA, and more conventional structures of CCL (Coupled Cavity Linac) type designed and realized to accelerate protons at energies gradually increasing up to $150 \mathrm{MeV}$.

The proton energy that the TOP IMPLART machine can deliver increases proportionally with the increase in the number of accelerating structures accelerators mounted in series.

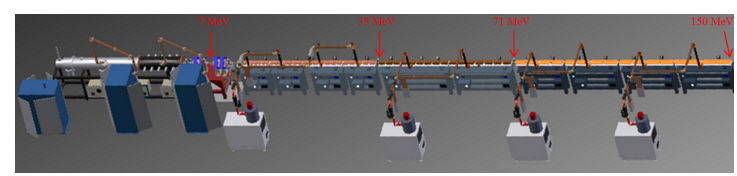

Figure 1. Layout of the TOP IMPLART accelerator

The intrinsic modularity of this linear accelerator gives the possibility to use the proton beam during the phase of assembling and also to test at energies lower than the values useful for clinical applications. This paper describes some experimental applications of the medium energy proton beams extracted by the TOPIMPLART accelerator in association with the operative and dosimetric controls implemented.

\section{THE TOP-IMPLART MACHINE}

The TOP IMPLART machine is made up of three sections: the low, the medium and the high energy lines. The accelerating sequence starts with a duoplasmatron ion source whence the extracted proton beam is sent into the RFQ and then speeded up in the DTL, both operating at $425 \mathrm{MHz}$. The current provided by the injector is continuously controlled up to $2 \mathrm{~mA}$ varying the voltage on an einzel lens placed after the ion source.

The injector output of $7 \mathrm{MeV}$ forks into two parts. A branch is composed of a transport line in which the particle beam is magnetically deflected upwards. The extraction point on the vertical line is located at about 2.5 meters above the ground and it allows irradiations in the 3-7 MeV energy range. The other branch heads the medium energy acceleration line working at $3 \mathrm{GHz}$ and composed by SCDTL modules, each having variable lengths (between 1 and 1.5 meter) and number of accelerating tanks (from 9 to 3 ). The system actually in operation consists of three SCDTL structures increasing the energy respectively to $11.6,18$ and 27 $\mathrm{MeV}$ (Figure 2). Two energies are presently available at the output of the horizontal line 27 and $18 \mathrm{MeV}$, achieved by switching on and off the last SCDTL module. The structure accelerating protons to $35 \mathrm{MeV}$ has been realized and is under commissioning. The four SCDTLs are actually powered by a single TH2090 klystron tube (15MW peak power, $15 \mathrm{~kW}$ average) installed into a pulse-forming network modulator 102 developed around 1990. The replacement with a TH2157A 10 MW peak power klystron and the K1 solid state modulator produced by SCANDINOVA is ongoing. The use of the new klystron/modulator system will allow the increase of the repetition rate presently limited to $25 \mathrm{~Hz}$ up to $100 \mathrm{~Hz}$.

This medium energy SCDTL chain will continue with 4 additional SCDTL modules up to $71 \mathrm{MeV}$ and the next high energy line will consist of $8 \mathrm{CCL}$ structures bringing the beam to the final energy.

The pulse length is variable from $15 \mu$ s to $80 \mu$ s for the injector and from $1 \mu \mathrm{s}$ to $4 \mu \mathrm{s}$ for the remaining accelerator environment. The total number of klystron is four: two in the medium energy section and two in the high energy part. The maximum accelerated current at $27 \mathrm{MeV}$ is $35 \mu \mathrm{A}$ in a $3.2 \mu \mathrm{sec}$ pulse.

The level of output current and charge are measured by different diagnostic devices: two Fast Current Transformers (FCT produced by Bergoz) [7] placed respectively at the entrance and the output of the SCDTL chain monitoring the current stability during the machine operation [8] and a thin ionization chamber, developed by ISS, placed at the exit of SCDTL-3. These tools are used to set the beam dose during the irradiation tests as will be described below.

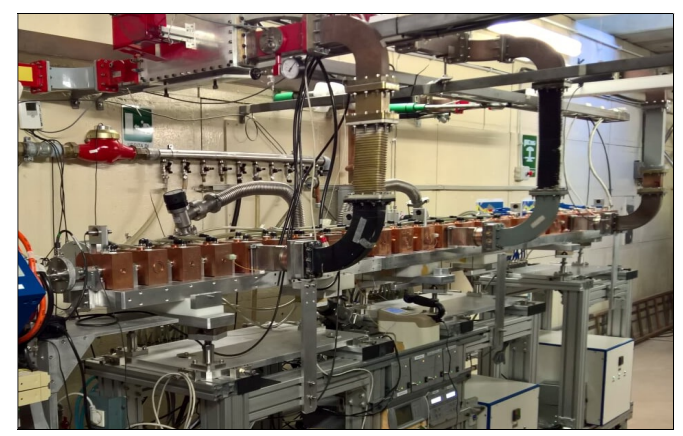

Figure 2. The horizontal line of the TOP IMPLART machine currently in operation

\section{IRRADIATION ACTIVITY}

The main characteristics of the TOP-IMPLART accelerator make it ready and adapted to the requirements of various proton experiments in different scientific disciplines.

The variable energy output and the pulsed time structure are very well suited for many applications and, thanks to the modularity of the linac, we can also make different types of irradiations with the medium energy beam (not available for clinical application) during the assembly steps of the machine: in this last year, we got the experience in animal radiobiology, proton exposure of biological material for space applications (irradiation in vivo of plants and animals), spectroscopy of X-ray induced by protons (PIXE analysis and PIXE differential of cultural heritage), dose calibration of radiotherapy monitors, test of innovative active dosimeters, measures of secondary radiation proton induced - passive and active measures of neutron fields [9, 10], development and characterization of passive dosimeters [8]. 
Four different setups are used corresponding to four different irradiation points depending on the samples size to be irradiated: two for the horizontal line (HL) and two for the vertical line (VL).

Along the HL, samples can be placed just after the exit in air of the proton beams, and here the vacuum seal window is made of $\mathrm{Ti}, 50 \mu \mathrm{m}$ thick. This irradiation point is useful for small size samples (high energy PIXE [11]) with the beam spot of $1.6 \mathrm{~mm} \times 1.2$ $\mathrm{mm}$ (rms values).

The second block of irradiation is located one meter from the exit window - here the beam passes through a volume of air that generates a spot-widening up to about $19 \mathrm{~mm}$ (rms) with a uniformity better than 95\% on a circle with $10 \mathrm{~mm}$ diameter, then collimated as required by the specific irradiation [12]. In this point, the beam energy drops from 27 to $24 \mathrm{MeV}$, corresponding to $6 \mathrm{~mm}$ of penetration depth in the water. The two HL irradiation points are shown in Figure 3 .

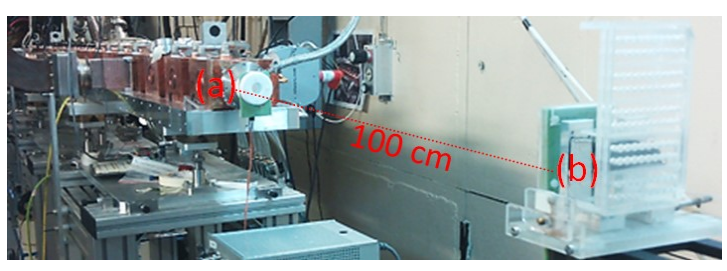

Figure 3. The horizontal line irradiation points of the TOPIMPLART accelerator: a) exit in air of the $27 \mathrm{MeV}$ proton beams, setup available for small size experiments; b) irradiation point $100 \mathrm{~cm}$ far from block (a), setup available for large size experiments with $24 \mathrm{MeV}$ protons

In the VL devoted to low energy beam experiments (3-7 MeV), there are two irradiation points: the first at the exit of the 90 deg bending magnet which has been used for low energy PIXE irradiation of cultural heritage artifacts [13]; the second, used for "in vitro" radiobiology experiments, is obtained with an "ad hoc" pipe, $69 \mathrm{~cm}$ long, giving a circular beam spot $13 \mathrm{~mm}$ large. This last line is arranged with a $2 \mu \mathrm{m}$ thick $\mathrm{Au}$ scatterer positioned after an $\mathrm{Al}$ collimator $(2 \mathrm{~mm}$ in diameter) in order to make the beam uniform on the bottom of a special stainless still PETRI dish for liquid materials [14].

These two points are visible in the photos of Figure 4. Table 1 summarizes some experiments carried out in the four different irradiation points and the typical values of proton energies.

Table 1. List of the irradiation points, experiments and proton energies for single experiment type

\begin{tabular}{|c|c|c|c|}
\hline $\begin{array}{c}\text { Irradiation } \\
\text { line }\end{array}$ & $\begin{array}{c}\text { Beam } \\
\text { size }\end{array}$ & Experiment description & MeV \\
\hline \multirow{2}{*}{ Vertical } & small & - Low energy PIXE [13] & $3 \div 6$ \\
\cline { 2 - 4 } & large & - Cell irradiation [14] & 5 \\
\hline \multirow{3}{*}{ Horizontal } & small & - High energy PIXE [11] & 18 \\
\cline { 2 - 4 } & large & $\begin{array}{c}\text { - Small animals [12] } \\
\text { - Vegetables [15] }\end{array}$ & 27 \\
\hline
\end{tabular}

Table 2 lists the system used for beam monitoring during irradiation experiments. We focused on the ionization dosimetry using different and accurate compared methods [16]: ionization chambers (IC) measurements, EPR/Alanine dosimetry, MOSFET radiation sensor analysis, film dosimetry, semiconductor detectors and luminescent devices.

- The IC measurements are commonly performed to monitor the particle beam fluence. The system adopted at the TOP-IMPLART accelerator is a square strip detector, realized by ISS, with a particular control of the electrical signal generated by the collection of ions produced during the passage of the beam protons between the plates [17].

- Alanine dosimetry is based on the revelation, with electron spin resonance technique (ESR), of stable free radicals produced by ionising radiation in the alanine amino-acid. In our applications, we employed commercial alanine dosimeters of cylindrical shape (with a diameter of $4.9 \mathrm{~mm}$ and a thickness of $3 \mathrm{~mm}$ ) made up of alanine amino-acid (representing the radiosensitive part) for $96 \%$ in weight, and of polyethylene (which is a binder allowing the realization in tablets) for the remaining $4 \%$. The conversion of the measurement EPR in "dose in water" is carried through a calibration curve obtained by irradiating groups of dosimeters to different dose values in the chosen range [18]

- The Metal Oxide Silicon Field Effect Transistor, MOSFET, is the most recent development for in-vivo dosimetry. It was designed to be used as an electronic replacement for the conventional Thermo Luminescent Devices and Diodes, with the size advantages and fewer correction factors. The dosimeter is a small silicon chip $(1 \mathrm{~mm} \times 1 \mathrm{~mm})$ with an active area of $0.2 \mathrm{~mm} \times 0.2 \mathrm{~mm}$. Radiation absorbed by a MOSFET results in a permanent change in its threshold voltage due to radiation-generated charge trapped in the gate oxide. This change in the voltage is proportional to the absorbed dose and sensitivities are typically 1 to $3 \mathrm{mV} / \mathrm{cGy}$. We use commercial MOSFETs applied in electron-radiotherapy and calibrated with an AutoSense system TN-RD-57-30 in collaboration with IFO [19].

- Radiochromic films are typically used in clinical dosimetry. The most commonly used radiachromic film is the GafChromic ${ }^{\mathrm{TM}}$ film: it is a colorless film with a nearly tissue equivalent composition ( $\mathrm{H} 9.0 \%$, C $60.6 \%, \mathrm{~N} 11.2 \%$, and $\mathrm{O} 19.2 \%$ ) that develops a blue color upon radiation exposure. It also contains a special dye that gets polymerized upon the exposure to radiation. For this reason, the absorbed dose inside the polymer can be revealed by measuring the transmission of light through the film changed in density. We operate with two types of film to measure the dose and to verify the size and homogeneity of the area hit by the proton beams: EBT3 and HD-V2 films. In $\mathrm{EBT}_{3}$ film the radiosensitive monomer is hedged by a polyester layer [20]. Therefore, the measurements of low-energy beams were made with the HD-V2 film because having no protection layer it reveals also 3 $\mathrm{MeV}$ protons. 
M. Vadrucci et al., Irradiation activity..., Rad. Applic., 2017, 2, 2, 101-107

Table 2. List of the monitoring and

diagnostic tools depicted in this work

\begin{tabular}{|l|l|}
\hline Monitoring and Diagnostic tools & $\begin{array}{c}\text { Irradiation } \\
\text { line }\end{array}$ \\
\hline - Gafcrhomic HD-V2/EBT3 for beam & \\
positioning, size, uniformity and dose & $\mathrm{VL} / \mathrm{HL}$ \\
measurements & $\mathrm{VL} / \mathrm{HL}$ \\
- Beam current measurements & $\mathrm{HL}$ \\
- FCT for current stability control & $\mathrm{HL}$ \\
- Thin ionization chamber for dose & $\mathrm{HL}$ \\
setting & $\mathrm{HL}$ \\
- EPR/Alanine dosimetry & $\mathrm{HL}$ \\
- MOSFET radiation sensor & $\mathrm{HL}$ \\
- Semiconductor dosimetry & $\mathrm{HL}$ \\
- Beam Micro Pattern Ionization & Chamber
\end{tabular}

- Finally, semiconductor detectors and luminescent devices are well suited to measure the beam energy, absorbed dose and dose equivalent, due to their small size that allows the excellent spatial resolution and the potential for real time read out [16].
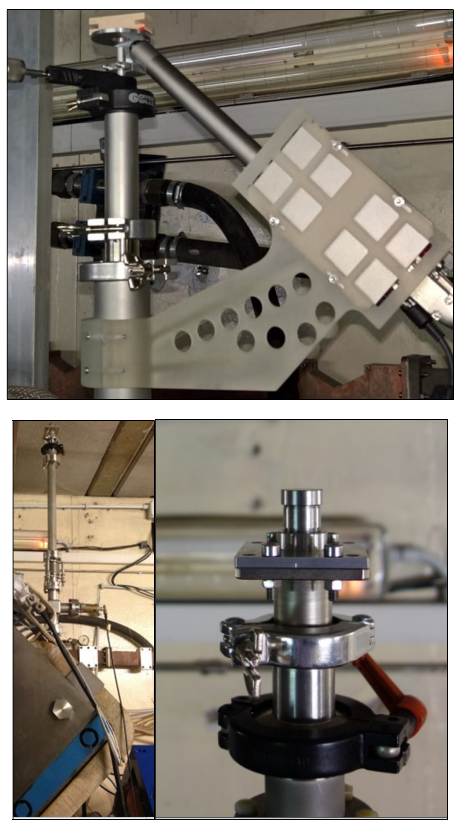

Figure 4. The vertical line irradiation points of the TOPIMPLART accelerator: the low-energy PIXE setup (top side); the in-vitro radiobiology setup (bottom side)

A systematic study based on the use of all these dosimeters has been done in the HL point in order to characterize the beam in terms of the overall stability, reproducibility in charge, dose, beam spot size and position. The measurements, done by comparing the results of the different dosimetric systems, showed reproducibility better than $3 \%$ for all the investigated parameters.

In the following, a brief description will be given on the irradiation activities conducted on VL for PIXE and PIXE-differential analysis, and on the HL for highenergy PIXE analysis in external beam setup and pilot experiments dedicated to a cosmic environment simulation program.

\section{Vertical line - Small beam}

The low-energy PIXE setup is used in the diagnostic activities with ionising radiation for the qualitative and quantitative elemental characterization, completely non-destructive and non-invasive of artistic finds in the framework of the ENEA-COBRA project [21].

The low-energy PIXE measurements were carried out placing the samples to be analyzed at the distance of $1 \mathrm{~cm}$ from the extraction window of the particles in air. The vacuum/air extraction window has been realized with a $7.5 \mu \mathrm{m}$ sheet of Upilex applied on a circular collimator, $300 \mu \mathrm{m}$ large, obtained with graphite titanium coated not to contaminate the X-ray field output.

The RX detector used is a KETEK GmbH mod. AXAS-A -X2614 with Silicon Drift Detector having a $500 \mu \mathrm{m}$ active layer and $65 \mathrm{eV}$ FWHM energy resolution at $5.9 \mathrm{keV}$ ( $\mathrm{Ka}$ line of $\mathrm{Mn}$ ) with $1 \mu \mathrm{s}$ shaping time. This sensor is $1.5 \mathrm{~cm}$ far from the sample (Figure 5) and fixed to an arm supporting the system containing the electric signal-transfer-chain.

The current of the extracted protons is on-line measured (monitoring the current lost over the collimator calibrated with the signal picked up by a monitor placed in air $1 \mathrm{~cm}$ far) and checked with a Tektronix MDO3014 $100 \mathrm{MHZ}$ oscilloscope.

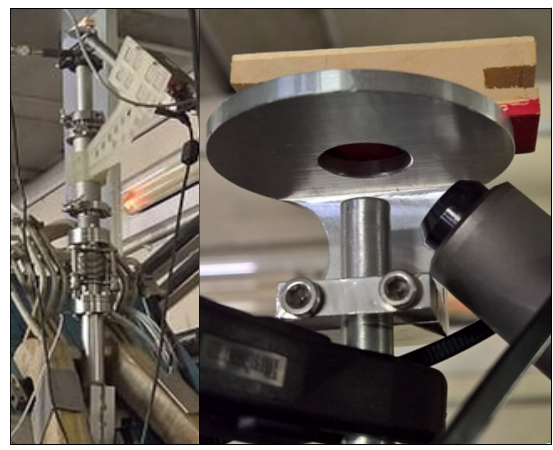

Figure 5. View of the setup developed for PIXE applications of the vertical line of the TOP IMPLART proton accelerator

(left). Vertical beam extraction detail with the sample holder and the X-ray detector (right)

The irradiated area of the sample is measured with HD-V2 GafChromic films. All materials to be irradiated are positioned on a rigid perforated sample holder support and all measurements were performed in air at room temperature (this is named external beam setup).

The differential PIXE measurements devoted to the stratigraphic analysis of the samples are carried out at different energies in the range $3-7 \mathrm{MeV}$ operating with the TOP-IMPLART injector turning on the single RFQ cavity for $3 \mathrm{MeV}$ protons and turning on also the DTL cavity to operate with energies up to $7 \mathrm{MeV}$.

The selection of the extracted energies is operated by the $90^{\circ}$ bending magnet acting as a spectrometer and guided by varying its supply current.

The energy attenuation and the spot size enlargement in air have to be considered: when protons cross the $1 \mathrm{~cm}$ long air column their energy reduces from the selected $3,4.5,6.1 \mathrm{MeV}$ to $2.75,4.32$, 
$5.85 \mathrm{MeV}$ respectively and the target area, measured by HD-V2, reaches 700 microns (a suitable size to characterize samples of cultural interest since in areas less than 1 millimeter the coatings of the works are more or less constituted by homogeneous layers of the same material although multi-elemental).

The analysis of the characteristic X-rays emitted from the target was carried out with GUPIX software (including specific spectral libraries and cross sections of radiation-matter reaction) using the remaining argon peak area for the acquired spectra normalization.

\section{Horizontal line - Small beam}

The setup of high-energy PIXE measurements is arranged along the HL accelerator output, as along the $\mathrm{VL}$, in the proximity of the beam exit in the air to avoid important variations of energy of the proton beams or the contamination of X-rays produced in the sample.

Figure 6 shows the external beam set up for irradiation of ancient Roman coins with $18 \mathrm{MeV}$ protons. This energy has been selected considering the penetration depth of the beam, the reaction cross sections of the protons with the elements constituting the specific target and considering the minimum energy of the X-rays emitted from the inner layers and that have to reach for the detector. The output current and charge are measured by the second FCT of the HL (as described above), the collimator applied at the end of the acceleration line has an aperture of $1 \mathrm{~mm}$ and the shape of the invested sample is measured with EBT3 films.

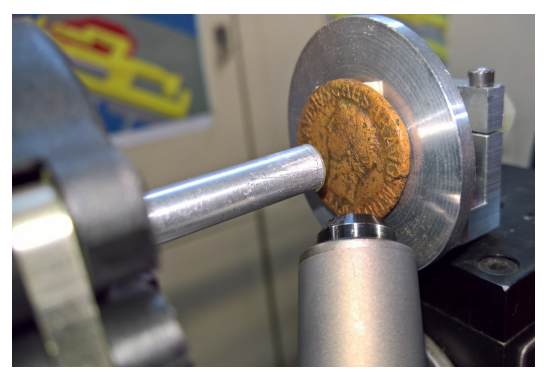

Figure 6. $18 \mathrm{MeV}$ PIXE setup of the TOP-IMPLART accelerator: horizontal beam extraction detail with the sample and the X-ray detector

The PIXE experience conducted in the framework of the COBRA project demonstrated the feasibility of a pulsed proton accelerator modulable in energy for stratigraphic surface and elemental analysis.

We carried out studies of qualitative and quantitative elemental identification of pigments on gypsum, ceramic and metallic materials. We found good response comparing our analyses with literature data and with other techniques conventionally used in the diagnosis and preservation of cultural heritage [11, 13].

Differential PIXE analyses are made by varying the incident energy of the protons and thus their penetration into the material. If the ratio between the yields of X-ray emission of the recognized elements, normalized to the incident protons, is independent from the proton energy then the elements are present in the same layer. If , on the contrary, the ratio between the yield of X-ray emission increases with the energy scale up of the incident protons, then the elements are contained in different layers and this means that several covers of the artefact occurred (for the realization or restauration).

\section{Horizontal line - Large beam}

The straggling of the $27 \mathrm{MeV}$ protons extracted in the air allows energy and geometrical changes $1 \mathrm{~m}$ far from the accelerator exit: the beam hit the target with $24 \mathrm{MeV}$ on a much larger area of the initial spot size. The measures carried out with alanine and radiochromic films reveal that, at $1 \mathrm{~m}$ from the beam exit, the window is still homogeneous over $95 \%$ in a circular area $1 \mathrm{~cm}$ large. This condition allows the irradiation of large samples of this size with the required uniformity without scanning systems. The dose value is set by reading the pulse output charge on a calibrated thin ionization chamber developed by ISS (Istituto Superiore di Sanità, Roma) placed at the output of SCDTL-3: operating at a bias voltage of $200 \mathrm{~V}$ and provided with electrodes realized with the aluminated mylar ( $12 \mu \mathrm{m}$ mylar, $4 \mu \mathrm{m}$ aluminum) $2 \mathrm{~mm}$ spaced: when the total output charge reaches a predefined value corresponding to the desired dose, the $\mathrm{RF}$ power in the $3 \mathrm{GHz}$ accelerator is switched off. In such way, it is possible to control the output charge during the irradiation with a precision of $0.5 \%$ independently by charge fluctuations during the pulse. The typical pulsed charge is around $10 \mathrm{pC}$. This configuration is used, for example, for irradiation of organic material (Figure 7) in the framework of the ENEA-ASI BioXtreme program $[15,22]$ : proton irradiation was performed to stress the on-board vegetables equipment for space flights to mimic the exposure of cosmic ionising radiations, mainly composed of protons.

Selected vegetables were fortified through specific biotechnology to better resist the extreme physical conditions that will be subjected when carried into the international space station [23].

Irradiation of hairy roots of the selected microtomato plants (a dwarf specimen), contained in PETRI dishes, have been made with medium-energy protons. The treatment doses are 0.5, 5 and 10 Gy to make a comparison with $\mathrm{X}$-rays and gamma rays exposures reported in the literature. For each dose value, 3 grams of biological material, contained in dishes of 0.33 grams and $5 \mathrm{~cm}$ in diameter, have to be irradiated. The irradiation setup for these samples involves a scanning system guided by an IPSES_MT2-Box controller appropriately shielded and operated with a specific software. The growth of the treated material is monitored in all of its phases: the development of particular components and the verification of the strengthening of specific properties is checked. The micro-tomato hairy roots are then subjected to the differential proteomics analysis by the extraction and fluorescent labeling of proteins separated on a 2D-gel and evaluated with bio-informatic methods to identify differential proteins obtained as proton treatment effect. 
M. Vadrucci et al., Irradiation activity..., Rad. Applic., 2017, 2, 2, 101-107

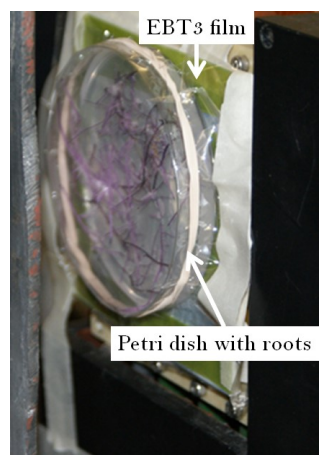

Figure 7. Setup used for vegetable irradiation experiments

As part of this same program, using the same geometrical setup and collimating, the beam with a specially made aperture of $1 \mathrm{~cm}$, the $27 \mathrm{MeV}$ proton beams performed in-vivo irradiations on specific anatomical districts of small animals (Figure 8). The aim of these experiments is to verify the radiobiological effects of the delivered dose to healthy individuals exposed to radiations during very long periods in space. Preliminary treatments were carried out to investigate the outcomes of the local exposure to $2 \mathrm{~Gy}$ of integral dose absorbed by $24 \mathrm{MeV}$ proton beams on spleen of anesthetized mice. After the treatment, spleen cells were collected, counted, stimulated and analyzed for statistical comparisons.

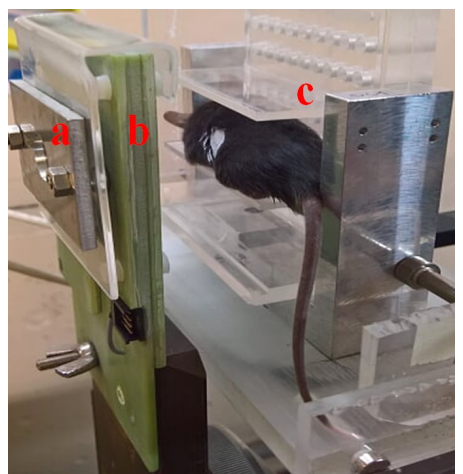

Figure 8. Setup of small animals' irradiation: view of the beam collimator (a), the ionization chamber for the dose delivery control (b) and mouse holder (c).

The irradiation campaign with protons in the framework of the BioXtreme program allowed us to collect interesting data and observations on the proteomics of plants and the immune system stimulation on the animals.

Acknowledgement: The paper is a part of the research done within the TOP-IMPLART project conducted in collaboration between ENEA, ISS and IFO and funded by the Regione Lazio. The authors would like to thank $M$. Chiari and A. Mazzinghi of INFN-LABEC of Florence for their support with PIXE measurements; E. Benvenuto, S. Massa, A. Desiderio, $C$. Pioli and F. Novelli for the Biotechnology and biomedical activities in the framework of the
BIOXTREME project partially supported by the Italian Space Agency.

\section{REFERENCES}

1. Particle therapy facilities in operation, Particle Therapy Co-Operative Group, Villigen, Switzerland, 2017.

Retrieved from:

https://www.ptcog.ch/index.php/facilities-in-operation Retrieved on: Feb. 5, 2017

2. G. Messina, U. Bizzarri, C. Ronsivalle et al., "The activity on accelerators at the ENEA Frascati Center:status and perspectives," in Proc. I European Particle Accelerators Conference (EPAC88), Rome, Italy, 1988, pp. 1477 - 1479

Retrieved from:

http://accelconf.web.cern.ch/AccelConf/e88/PDF/EPA C1988 1477.PDF

Retrieved on: Feb. 5, 2017

3. L. Picardi, G. Messina, C. Ronsivalle, A. Vignati, "The activity on linear accelerators at the ENEA Frascati Center," in Proc. 1992 Linear Accelerator Conference, Ottawa, Canada, 1992, pp. $510-512$

Retrieved from:

http://accelconf.web.cern.ch/AccelConf/l92/papers/tu 4-69.pdf

Retrieved on: Feb. 5, 2017

4. L. Picardi, C. Ronsivalle et al., "The italian IORT project," in Proc. EPAC 2000 Conference, Vienna, Austria, 2000, pp. 2545 - 2547

Retrieved from:

http://accelconf.web.cern.ch/AccelConf/eoo/PAPERS/ WEP5B04.pdf

Retrieved on: Feb. 5, 2017

5. C. Ronsivalle et al., "The TOP-IMPLART Project," Eur Phys. J. Plus, vol. 126, no. 68, pp. 166 - 168, Jul. 2011. DOI: $10.1140 /$ epjp/i2011-11068-x

6. W. D. Kilpatric, "Criterion for Vacuum Sparking designed to include both rf and dc," Rev. Sci. Instrum., vol. 28 , no. 10, p. 824, 1957 .

DOI: $10.1063 / 1.1715731$

7. C. Ronsivalle et al., "The TOP-IMPLART linac: machine status and experimental activity," in Proc. IPAC17, Copenhagen, Denmark, 2017, pp. $4669-4672$ Retrieved from:

http://accelconf.web.cern.ch/AccelConf/ipac2017/pape rs/thpvaogo.pdf Retrieved on: Aug. 5, 2017

8. M. Vadrucci et al., "First beam characterization of the TOP-IMPLART proton linear accelerator for cancer radiotherapy," in Proc. 55 $5^{\text {th }}$ Annual Meeting for the PTCOG 2016, Prague, Czech Republic, 2016, pp. 201 202

DOI: 10.14338/IJPT.16-PTCOG-1.1

9. M. Vadrucci, P. Ferrari et al., "Preliminary characterization of the neutron field in TOP-IMPLART proton therapy facility," presented at the $56^{\text {th }}$ Annual Meeting for the PTCOG, Kanagawa, Japan, 2017. DOI: 10.14338/IJPT.17-PTCOG-1.1

10. P. Ferrari, M. Vadrucci et al., "Preliminary study of neutron field in TOP-IMPLART proton therapy beam," to be published in Proc. NEUDOS 2017, Kraków, Poland, 2017

11. M. Vadrucci et al., "Analysis of Roman Imperial coins by combined PIXE, $\mu$-XRF and LIBS techniques," Microchem. J., no. spec. issue, to be published.

12. F. Novelli, M. Vadrucci et al., "Effects of in vivo proton irradiation on mouse spleen cells," Radiation and Applications vol. 2, no. 3, to be published.

13. M. Vadrucci et al., "A new small-footprint externalbeam PIXE facility for cultural heritage applications 
using pulsed proton beams," Nuclear Instruments and Methods in Physics B, to be published.

14. M. Vadrucci et al., "The low-energy proton beam for radiobiology experiments at the TOP-IMPLART facility," Biophysics and Bioengineering Letters, vol. 8, no. 1, May 2015.

Retrieved from:

http://ojs.uniroma1.it/index.php/CISB-

BBL/article/view/13287/13086

Retrieved on: Aug. 5, 2017

15. M. Vadrucci et al., "Mimicking extreme astrophysical environments: first trials of irradiation of plant tissues with the TOP-IMPLART protontherapy accelerator," presented at the Agrospace 2016 Conferenece, Sperlonga, Italy, 2016

16. M. Vadrucci et al., "Diagnostics methods for the medium energy proton beam extracted by the TOP IMPLART linear accelerator," in Proc. IPAC 2017, Copenhagen, Denmark, 2017, pp. 4673 - 4675

Retrieved from:

http://accelconf.web.cern.ch/AccelConf/ipac2017/pape rs/thpva091.pdf

Retrieved on: Aug. 5, 2017

17. E. Cisbani et al., "Micro pattern ionization chamber with adaptive amplifiers as dose delivery monitor for therapeutic proton LINAC," in Proc. IBIC 2016, Barcelona, Spain, 2016, pp. $464-467$

Retrieved from:

http://accelconf.web.cern.ch/AccelConf/ibic2016/pape rs/tupg51.pdf

Retrieved on: Aug. 5, 2017

18. C. De Angelis et al., "Characterization of a $27 \mathrm{MeV}$ proton beam linear accelerator," to be published in Proc. NEUDOS 2017, Kraków, Poland, 2017
19. M. D. Falco et al., "Characterization of a cable-free system based on p-type MOSFET detectors for "in vivo" entrance skin dose measurements in interventional radiology," Med Phys, vol. 39, no. 8, pp. $4866-4874$, Jul. 2012.

DOI: $10.1118 / 1.4736806$

PMid: 22894413

20. M. Vadrucci et al., "Calibration of GafChromic EBT3 for absorbed dose measurements in $5 \mathrm{MeV}$ proton beam and 6oCo $\gamma$-rays," Med. Phys., vol. 42, no. 8, pp. 467884, Aug. 2015.

DOI: $10.1118 / 1.4926558$

PMid: 26233195

21. ENEA-COBRA project, Development and dissemination of methods, advanced technologies and tools for the conservation of cultural heritage, based on application of radiation, ENEA, Rome, Italy Retrieved from: http://cobra.enea.it/english Retrieved on: Jul. 10, 2017

22. M. Vadrucci et al, "Proton irradiations of micro-tom red hairy roots to mimic space conditions," in Proc. IPAC2015, Richmond (VA), USA, 2015, pp. 2249 2252

Retrieved from:

http://accelconf.web.cern.ch/AccelConf/IPAC2015/pap ers/tupwio05.pdf Retrieved from: Aug. 5, 2017

23. S. Massa et al., "Tomato hairy root cultures as a platform for the bioproduction of valuable molecules and as a tool to test extreme astrophysical conditions," presented at the Agrospace 2016 Conferenece, Sperlonga, Italy, 2016 